\title{
What if I had a third arm? An EEG study of a supernumerary BCI system
}

\author{
Jaime A. Riascos ${ }^{\mathrm{a}, \mathrm{b}, \mathrm{c}}$, David Steeven Villa ${ }^{\mathrm{c}, \mathrm{d}}$, Anderson Maciel ${ }^{\mathrm{c}}$, Luciana \\ Nedel $^{\mathrm{c}}$, Dante Barone ${ }^{\mathrm{c}}$ \\ Porto Alegre, Brazil. \\ ${ }^{a}$ Autonomous University Corporation of Nariño - Pasto, Colombia. \\ ${ }^{b} S D A S$ - Research Group. \\ ${ }^{c}$ Institute of Informatics - Federal University Rio Grande do Sul (UFRGS) - Porto \\ Alegre, Brazil. \\ ${ }^{d}$ Ludwig-Maximilians-Universität München (LMU Munich) - Munich, Germany.
}

\begin{abstract}
Motor imagery Brain-Computer Interface (MI-BCI) enables bodyless communication by means of the imagination of body movements. Since its apparition, MI-BCI has been widely used in applications such as guiding a robotic prosthesis, or the navigation in games and virtual reality (VR) environments. Although psychological experiments, such as the Rubber Hand Illusion - RHI, suggest the human ability for creating body transfer illusions, MI-BCI only uses the imagination of real body parts as neurofeedback training and control commands. The present work studies and explores the inclusion of an imaginary third arm as a part of the control commands for MI-BCI systems. It also compares the effectiveness of using the conventional arrows and fixation cross as training step (Graz condition) against realistic human hands performing the corresponding tasks from a first-person perspective (Hands condition); both conditions wearing a VR headset. Ten healthy subjects participated in a two-session EEG experiment involving open-close hand tasks, including a third arm that comes out from the chest. The EEG analysis shows a strong power decrease in the sensory-motor areas for the third arm task in both training conditions. Such activity is significantly stronger for Hands than Graz condition, suggesting that the realistic scenario can reduce the abstractness of the third arm and improve the generation of motor
\end{abstract}

\footnotetext{
${ }^{*}$ Corresponding author: jarsalas@inf.ufrgs.br
} 
imagery signals. The cognitive load is also assessed both by NASA-TLX and Task Load index.

Keywords: Motor Imagery, Brain-Computer Interface, Rubber-Hand Illusion, Embodied Cognition.

\section{Introduction}

The primary purpose of Human-Computer Interaction (HCI) is seeking

new alternatives for communicating humans and machines, and this effort is more evident when users with motor disabilities show difficulties using standard interfaces [1]. Brain-Computer Interface (BCI) is the technology that enables bodyless communication with machines or devices; this is done using the translation of brain signals into command outputs [2].

BCI commonly employs the electrical activity in the brain (EEG) elicited during a specific task. Depending on the nature of this activity, BCI is characterized as passive, active or reactive [3]. Passive systems use signals that arise without voluntary control. It is used fundamentally to asses mental states and enhance the human-computer interaction [4]. Active BCI works with the self-induced brain activity produced by the user independently of external events. It has been used as a control signal [5]. Finally, reactive BCI relies on the signals elicited by the reaction to specific external stimuli, which could be used to control an application as well [6].

Since the activation patterns of imaginary body movements involves both brain regions (sensory and motor areas) and neural mechanisms similar to the executed movement [7], the Motor Imagery BCI (MI-BCI) has been widely used and explored in active BCI [8]. MI-BCI employs the amplitude changes voluntarily elicited by the mental rehearsal of physical motor actions. Such variations are known as event-related de-synchronization and synchronization (ERD/ERS). These patterns have been successfully used for studying the neural mechanisms associated with motor actions, as well as a feature for classification in motor-related BCI systems $[9,8,10,1]$.

Despite BCI being a promising and useful application, there are still several challenges to be addressed. Chavarriaga et al. [11] discuss concrete research avenues and guidelines to overcome common pitfalls in BCI. Their paper is the outcome of a meeting held at the workshop "What's wrong with us? Roadblocks and pitfalls in designing BCI applications". They summarize four main topics that influence any closed-loop BCI system: 
a) Signal processing and decoding: the signal processing of EEG data, and consequently BCI systems, is boosted by the fast growth of machine learning and unsupervised systems (i.e., deep learning) [12].

b) End Users: the creation of objective either questionnaires or pretests to identify potential user should be considered prior to a BCI implementation.

c) Performance metrics and reporting: BCI's metrics are a topic under discussion [13] since the classification accuracy is not enough for evaluating BCI systems, the creation of new metrics becomes fundamental [14].

d) Feedback and user training: several efforts have been made in order to include the user inside the BCI loop [15], creating affordable and intuitive interfaces, considering human factors on their design.

In effect, immersive technologies have recently played an essential role in overcoming the feedback and user training challenge. Among them, Virtual Reality (VR) is one of the most promising technologies, giving the users a sensation of actual presence in virtual worlds. VR has been effectively used in several areas, from health-care for rehabilitation and training [16] up to data visualization and serious games $[17,18]$. Likewise, VR has been used in BCI for a visual presentation feedback of the current task carried out by the user. Lécuyer et al. [19] discuss some of the current applications developed using BCI with VR, namely MindBalance [20], Simulation of wheelchair control [21], and "use the force" [22]. These studies, as highlighted by the authors, show the successful use of VR with BCI.

Another important thing about MI-BCI applications is that, so far, they have essentially used attached body parts. In other words, MI-BCI focuses on mental representations of jointed limbs following the human anatomy constraints (e.g., two arms, two legs, two feet, in a symmetrical distribution). To the authors' knowledge, nevertheless, there are neither explorations nor applications that include non-embodied human limbs in BCI systems, even though Rubber Hand Illusion (RHI) experiments demonstrated the human capabilities to create body transfer illusions [23, 24]. Indeed, RHI does not only demonstrate a static body illusion representation (sense of ownership), but also an active movement eliciting a body illusion (sense of agency) [25]. 
In that vein, this paper presents the complementary results regarding the inclusion of a third arm as a control command in a BCI system: an EEG analysis of the induced brain oscillatory activity elicited by the third arm using Event-Related Spectral Perturbation (ERSP). A preliminary study addressed an offline exploration of the classification of the third arm task [26]. Continuing with that study, throughout this research, we compared the approach under two training conditions: the conventional Graz paradigm (cross and arrows) and immersive human-like feedback. Moreover, we included a cognitive load assessment by both the subjective questionnaire (NASA-TLX) [27] and the Task Load Index using EEG data [28]. Finally, we used the Movement Imagery Questionnaire - 3 (MIQ - 3) [29] before the experiment to assess the movement imagery ability of the users. The findings suggest that ERS/ERD patterns are elicited by the virtual third arm. Moreover, in line with the literature, the realistic training enhances the modulation of such patterns but creating an additional cognitive load (presumably caused by the visual processing).

The remainder of this paper is structured as follows: section 2 presents the state-of-art in BCI, applications that use either VR or body illusions. Then, section 3 shows the materials, methods, and details of the experimental procedure. Finally, section 4 provides the main findings that are discussed in section 5, and section 6 presents the concluding remarks.

\section{Related Works}

Virtual Reality is a powerful tool to improve the BCI training and enhancing the feedback experiences [30]. The learning task should include an intuitive feedback so that the users can easily understand the action to be executed and improve their performance. However, it is currently hard to choose the right feedback presentation, and it should be a motivating and engaging environment [11], besides being natural and realistic. Here, VR can be shown as a real alternative for tackling the feedback presentation issue.

Lotte et al. [31] show how combining BCI with VR can carry towards a new and improved BCI system. Nevertheless, such VR feedback can also introduce some interference to the motor imagery-related brain activity used by the BCI because both $\mu$ and $\beta$ bands are reactive in motor imagery and observation of the real movement [9]. An interesting study carried out by Neuper et al. [32] explores the influence of different types of visual feedback in the modulation of the EEG signal during the BCI control. Using a video 
to show a first-person view of an object-directed grasping movement, they were able to found modulation activity in sensorimotor rhythms caused by this real feedback stimulus. They highlight the importance of the amount of information provided by this condition in order to reduce the reactive bands.

Ron-Angevin and Diaz-Estrella [33] made a first comparison between the screen condition (Graz) and VR in a BCI scenario, focusing on the performance (classification rates). They successfully found improvements in the feedback control of the VR condition in untrained subjects. However, they used car navigation as a task, which can be seen as unnatural and abstract when compared to an embodied experience. The studies cited above have used different feedback stimuli, but none of them has used a virtual human avatar, which could be useful for the training step. Recently, Skola and Liarnokapis [34] addressed such problem comparing the Graz paradigm against a human-like avatar performing the user's motor actions synchronously. The authors report improvements in both ERD/ERS modulation and classification rates by the neurofeedback-guided motor imagery training. Likewise, Braun et al. [35] report the same sort of results using an anthropomorphic robotic hand as a visual guide. Also, they found differences between the two conditions in the electrodermal activity and subjective measures. Both works reported that they were inspired by the RHI. They also include within their discussions, the analysis of sense of ownership, agency, and self-location towards the non-body object, concepts that are being recently taken into account in BCI research [36, 37].

Although, from the RHI theory, it is demonstrated that the body transfer illusion can be effectively used with non-attached limbs in both passive (presence) and active (movement) conditions [25]. Up to this point, supernumerary limbs BCI system had not been approached. Bashford and Mehring [38] proposed this possibility with their work. They used an imaginary third arm for assessing the ownership and agency of a non-body limb in an imitation BCI (i.e. subjects think that their EEG activity is controlling the arm). Results show that there is independent ownership and control - based on the correct movements observed against the subject movements - of the third arm keeping the sense of ownership of the real hands. These findings suggest the capabilities of human of extrapolating limbs to execute motor actions. However, they did not study the use of this third arm as a control command inside the BCI loop. A recent work proposed by Song and King [39] demonstrates that using an RHI-based paradigm can significantly enhance the MI signals for BCI systems. 
The paper's contribution includes a step towards the creation of supernumerary MI-BCI systems. Here, we performed an EEG study of the user's ability to imagine a third imaginary arm in a BCI paradigm. We also compared the effectiveness of using the conventional arrows and fixation cross as training step (Graz) against a first-person view using a human avatar (Hands). Both training conditions were carried out in a VR environment.

\section{Materials and Methods}

\subsection{Overview}

An offline MI-BCI experiment, which uses EEG for recording the data and VR scenarios for presenting the stimulus, was conducted in a reduced noise room. The experiment's aim is to study the feasibility of including a virtual third arm in a MI-BCI system while the traditional training paradigm (Graz) is compared against a first-person view using a human avatar. There were two recording sessions with two runs in each one with a resting time between them. The sessions were conducted on two separate days within one week. Only on the first day, the participants had to fill up three questionnaires: MIQ-3, demographics and Edinburgh Handedness. Likewise, after each session, participants filled the NASA-TLX form.

\subsection{Participants}

Ten right-handed volunteers (four women) participated in the study. Participant ages were within 18 and 34 years old with a mean of 23. All participants had basic informatics knowledge. Only 30\% did not have previous experience with VR and no one had any previous experience in MI-BCI. No one had problems with head movements. Half of the population had visual impairments (mainly myopia and astigmatism) and used glasses to reduce them. The experiment was conducted in accordance with the Declaration of Helsinki. Participants were informed both oral and writerly about the procedure and the EEG recording. All participants gave written informed consent.

\subsection{VR Scenarios}

For the VR exposition, we used a head-mounted display (HMD) Oculus Rift CV1 with a resolution of $2160 \times 1200$ (1080 x 1200 per eye), refresh rate of $90 \mathrm{~Hz}$, a $110^{\circ}$ field of view, and both rotational and positional tracking to render the immersive scene. We used the popular game engine Unity3D 
to develop the immersive scene that was intended to assist the users when imagining and performing motor actions with their left and right real arms and the middle imaginary one (see the top of Figure 1).

There was a special focus on the realism of the models: left and right hands were placed matching with the rest positions of the real hands. A third hand was placed in the middle of the body, like emerging from the chest trying to avoid visual relations with the left or the right arm. The fingers on the third arm also were modified to be symmetric. In this sense, since that the thumbs in either left and right hand can indicate to which arm it belongs, their were removed from the third arm. Thus, it is identified as an independent arm and not a copy or extension of the existing arms. Highquality textures were used with shaders designed to highlight generic skin details. Bones in each finger preserve the average human hand proportions.

\subsection{Experimental Procedure}

The subjects sat comfortably in an armchair and were asked to rest their arms in the armrests and avoid any other movements during the recordings. Initially, the participants wore the HMD for getting into the scene and running several trials for learning the instructions previously read. After the training, we mounted the EEG cap followed by the traditional gelling process, and then we fit the HMD. We tried as much as possible to avoid that the HMD frame touches the EEG electrodes. Moreover, we checked the signal quality before and after mounting the HMD to detect any avoidable interference.

The experiment involves the execution of four different tasks in two experimental conditions. The subjects were invited to rest (RS), or to move a specific hand: third hand $(\mathrm{TH})$, left hand $(\mathrm{LH})$, and right hand $(\mathrm{RH})$. Conditions considered were Graz, and Hands. The Hands condition involved the presentation of a human-like avatar (see the top of Figure 2), whereas Graz the presentation of arrows (see the middle of Figure 2).

The two experimental conditions followed the timing protocol proposed by Pfurtscheller [9]. The users performed 20 trials of each task randomly selected (described below) with a duration of 7 seconds each (see the bottom of Figure 2). The main difference between the conditions lies in the visual feedback, as follows:

a) Graz condition: starting with a gray screen (resting state), at time $2 \mathrm{~s}$, a fixation cross at the center of the scene was displayed with a short 
warning tone ('beep') which indicates to the user to pay attention to the incoming visual cue presented at time $3 \mathrm{~s}$. At time $4 \mathrm{~s}$, the user had to perform the motor task for three seconds. The color of the arrows indicates the task (red for execution and white for imagination) and the direction indicates if the hand should be either left or right. The third arm cue was an arrow pointing upwards (see the middle of Figure $2)$.

b) Hands condition: at the start, the user's hands were placed in the equivalent real arms positions (resting state), at time $2 \mathrm{~s}$, the same auditory cue starts indicating an incoming stimulus. Next at time 3s, a visual cue is introduced without animation to let the users to be prepared for the action they will perform. At time $4 \mathrm{~s}$, the animation is introduced, and the user must perform either the mechanic or imaginary operation. This state continues until the end of the task (three seconds more). As for the visual cues, the real skin shading represents actual open-close hand movements, while transparent shading represents imaginary movements. Moreover, it is important to highlight that the third arm appears in the scene only when this specific trial is necessary. In other trials, there are just two visible hands (see the top of Figure 2).

Following [40], subjects were instructed to perform the kinesthetic experience during the execution of motor imagery tasks, i.e., imagining the sensation of performing the motor tasks rather than the visual representation of the movement. The authors suggest that kinesthetic motor imagery is essential to elicit sensorimotor patterns (ERD $\backslash S)$. Besides this, in order to avoid the carry-over bias, both experimental conditions were counterbalanced across participants (i.e. five subjects start with Hands condition and the rest with Graz). Likewise, it is necessary to mention that the movement animations were applied directly to the bones always looking for a natural behavior of the hand. The animations are predefined, they are not based on the user's EEG activity or motion.

Finally, in contrast to Skola and Liarnokapis [34] where the Graz condition is presented in a monitor, we made comparisons of the Graz and Hands conditions in an immersive virtual environment. Therefore, the users have to wear the HMD in both conditions. The background of Graz scenario was set to gray, avoiding high contrast that could produce discomfort on the user's eyes. 


\subsection{Data Acquisition}

We collected the EEG data using an OpenBCI 32 bit board at a sampling rate of $250 \mathrm{~Hz}$. Following the 10-20 EEG placement system, eight passive gold cup electrodes were used and placed at sensorimotor cortex (see the bottom of Figure 1), namely, frontal (F3, Fz, F4) central (C3, Cz, C4), and parietal (P4, P3) cortices. Left and right mastoids were used as reference and ground electrodes respectively. Labstreaminglayer (LSL) is used for recording and synchronizing the EEG data with the Unity trials through LSL4Unity (a third party software) [41].

\subsection{EEG signal processing}

We used EEGLAB (14.1) [42] (under Matlab 2017b) for processing the .XDF file created by LSL. Following the usual procedure for analysis motorimagery-related EEG patterns (sensorimotor rhythms) [43], we initially downsampled the signals at $115 \mathrm{~Hz}$ and band-passed at $1-35 \mathrm{~Hz}$ using a finite impulse response (FIR) filter. Later, we used the Cleanline plugin at 50-115 $\mathrm{Hz}$ instead of a notch filter to avoid band-holes, and distortions at the cutoff frequency. Likewise, we rejected bad channels (excluding the sensorimotor ones) using Cleanraw plugin. The rejected channels were then interpolated using a spherical function. Finally, we used the common average reference (CAR).

\subsection{Event-related spectral perturbation}

The event-related spectral perturbation (ERSP) is a generalization of the ERD/ERS patterns. ERSP computes the changes of the spectral powers in time-frequency domains, relative to the stimuli [44]. Thus, with this approach, the changes of the EEG signals elicited by motor imagery events can be detected alongside of the spectral band and epoch. ERSP values were computed for every mental task (TH, LH, RH, RS) in Graz and Hands conditions using the newtime function of the toolbox in the filtered data. We used a time window of $-500 \mathrm{~ms}$ to $2500 \mathrm{~ms}$, displayed between $5 \mathrm{~Hz}$ and $30 \mathrm{~Hz}$; Also, significant alpha was setup to 0.05. The sensorimotor area composed by the electrodes $\mathrm{C} 3, \mathrm{Cz}$ and $\mathrm{C} 4$ were used to display the time-frequency ERD/ERS maps (Figures 3 and 4).

\subsection{Task Load Index}

Besides the subjective assessment of the cognitive load by the NASATLX [27], we also used the Task Load Index (TLI) developed by Alan Gevins 
and Michael E. Smith [28] in order to have an objective measure of the task load. The authors found that the power changes of $\theta$ at frontal mid-line sites and $\alpha$ at parietal sites are related to the task load associated to the mental effort required for task performance. Thus, this index can be measured by the ratio of $\theta$ to $\alpha$. In this work, we used the spectopo function to calculate the average of the absolute power of frontal mid-line $(\mathrm{F} 3, \mathrm{Fz}, \mathrm{F} 4) \theta$ and parietal (P3-P4 plus Cz) $\alpha$ to assess the mental tasks per condition (Graz and Hands) as follows:

$$
T L I=\frac{\mu\left(\alpha_{F 3, F z, F 4}\right)}{\mu\left(\theta_{P 3, C z, P 4}\right)}
$$

\section{9. $M I Q-3$}

Despite Motor Imagery is a fundamental constructor of any healthy person, i.e., that all humans should have the capacity of imagining and planning motor activities, some people could face limitations to perform imaginary activities. In such vein, several questionnaires were made in order to subjectively assess the individual ability to perform imaginary motor tasks, such as the Vividness of Movement Imagery Questionnaire (VMIQ) [45] or Movement Imagery Questionnaire (MIQ) [46]. The MIQ-3, a recent version of the MIQ, and in different of the VMIQ, assesses three kinds of imagery [47]:

a) Internal Visual Imagery: visual image of the performed movement from an internal perspective (i.e., the subject performing and seeing the action from a 1 st person perspective).

b) External Visual Imagery: visual image of the performed movement from an external perspective (i.e., the subject performing and seeing the action from a from a 3rd person perspective)

c) Kinesthetic Imagery: creating the feeling of making the performed movement without actually doing it.

This survey is a 12-item questionnaire to asses the capacity to image four simple movements: a knee lift, jump, arm movement, and waist bend, in a scale from 1 (very hard) to 7 (very easy). The MIQ-3 demonstrated excellent psychometric properties, internal reliability, and predictive validity. This paper uses an adaptation of the MIQ-3 questionnaire to the Portuguese language [48]. 


\section{Results}

\subsection{ERSP maps}

Figures 3 and 4 show the time-frequency representation of significant (bootstrap method, $p<0.05$ ) ERD/ERS values (blue indicates ERD) for the Hands and Graz condition respectively. These maps come from a single subject $(6)^{1}$ at electrode positions $\mathrm{C} 3, \mathrm{Cz}$, and $\mathrm{C} 4$. The analysis of these maps reveals, certainly, the brain activity elicited by the imagination of hands movements (motor imagery), and that the third arm emerging from the chest can elicit similar patterns.

For the TH task, at C3 position in Hands condition, a strong power decrease is clearly visible around $500 \mathrm{~ms}$ after stimulus onset, and this behavior repeats in almost the whole frequency range. In the other two imagery tasks, LH has a decrease in Alpha followed by an increase in Alpha and Beta. RH has a similar pattern but without a clear ERS activity in alpha. Interestingly, TH task held the ERD activity during the rest of the epoch after $1000 \mathrm{~ms}$ with few ERS in middle and high beta bands. Conversely, in Graz condition at C3, the ERD patterns of the TH task are attenuated and widespread with some ERS activity at the end of the epoch in high Beta band.

At $\mathrm{Cz}$ in Hands condition, the TH task presents a few ERS activity that starts around $500 \mathrm{~ms}$ in Alpha, and an ERD that starts around $1000 \mathrm{~ms}$ in Alpha and Beta bands. LH presents a strong ERS activity in both Alpha and Beta anticipated by an ERS in Alpha and middle Beta. RH has a strong ERD activity in Alpha and Beta and posteriorly some ERS in high and low Beta. Meanwhile, in Graz condition, TH shows ERD patterns in Alpha until the first $1000 \mathrm{~ms}$. At the end of the epoch, some ERS activity is presented in high Beta. In LH, there is an ERD pattern in Alpha during the first 500ms and a widespread ERS activity later. RH holds the ERD in Alpha at the same time with some ERS in middle Beta.

Similarly, TH task in Hands condition presents an ERD pattern around $500 \mathrm{~ms}$ in Alpha and middle Beta at the C4 position. This activity is held again during the whole epoch (mainly in Alpha). Few ERS activity is found in high Beta after 1000ms. The ERS activity is most prominent in Alpha and low-middle Beta for LH, meanwhile, RH shows an ERD/ERS pattern in Alpha and Beta in the first 1000ms. For Graz condition, the ERD patterns

\footnotetext{
${ }^{1}$ In order to show visibly the phenomena, we used the EEG data from the subject who obtained the best classification rates [26].
} 
of TH task are widespread in Alpha and Beta between 500ms and 1500ms with some presence of ERS in high Beta. LH has a strong ERD activity during the first 1000ms in Alpha and some widespread ERS in high Beta. RH has strong ERD patterns during the same previous time in both Alpha and middle Beta followed by a strong ERS activity in Alpha, extended along of the epoch.

\subsection{Topographical Maps}

Figure 5 shows the representative set of topographical distributions of each mental task obtained from the same subject in Alpha and Beta bands for the first second after the cue. The TH task, for both bands in Hands condition only exhibits ERD activity (more prominent in Alpha band) mainly on the contralateral $(\mathrm{C} 3)$ and middle $(\mathrm{Cz})$ regions. On other hand, $\mathrm{TH}$ in Graz condition presents ERD/ERS activity in both bands; in effect, it can be seen a strong ERD on the frontal lobe (F3) and ERS on parietal region (P3). These findings could suggest that the brain activity elicited by the third arm is not only associated with sensorimotor areas, but also the imagination effort is visible at frontal and parietal regions (more clear in Hands condition for both bands).

\subsection{Power spectral analysis}

In order to explore the differences of the ERD/ERS patterns among tasks in the two conditions, Figures 6 and 7 show comparisons of the power changes of the TH task against the other imagery tasks (LH-RH) in both conditions using the same electrodes array from the same subject $(6)$. Blue lines represent $\mathrm{TH}$, the red ones LH while RH is represented by green lines. Moreover, Figure 8 presents the power comparison of the TH task in both conditions. Blue line indaicates The paired Wilcoxon signed-rank test was used to find out significant differences between conditions $(p<0.05)$. They are indicated by shaded blocks.

The differences presented by TH-LH and TH-RH are significantly more broad-banded at C3 than other channels in Hands condition (Figure 6). Meanwhile, Graz condition presents similar significant region sizes among the channels (Figure 7). At C3, both cases (TH-RH, TH-LH) in Hands condition show significant differences in almost the whole frequency range. Conversely, in Graz condition, TH-RH shows more significant differences in Alpha and low Beta than TH-LH, but they share the significant region around $20 \mathrm{~Hz}$ up to $25 \mathrm{~Hz}$. 
At $\mathrm{Cz}$ in Hands condition, the TH-LH comparison does not have a significant region in the Alpha band, but it shares a low and middle Beta with TH-RH, which has significant differences in Alpha and high Beta sub-bands. For the Graz condition in the same location, the TH-LH comparison indicates wide-spread sub-band regions for the Beta, in Alpha only a small region around $10 \mathrm{hz}$ is presented and, in the meantime, TH-RH shows a consistent region in Alpha and low and high Beta.

Finally, at $\mathrm{C} 4$ in Hands, the TH-RH comparison shows wider regions than TH-LH, especially in Alpha and middle Beta rhythms. The same behavior is presented in the Graz condition, where TH-RH has more significant regions in Alpha and low and middle Beta than TH-LH, which does not have a significant difference in Alpha, only in several sub-bands along Beta, mainly above than $15 \mathrm{~Hz}$.

In the comparison of the TH task between conditions (Figure 8), there is a stronger power decrease in Hands than in Graz condition, in line with the ERS/ERD maps (Figures 3 and 4). Such difference is more evident at C3 than the other channels. Likewise, C3 noticeably shows significant regions within both Alpha and Beta rhythms, whereas $\mathrm{Cz}$ is more often in middle and high beta, and $\mathrm{C} 4$ in Alpha and middle Beta.

\subsection{Cognitive Load and MIQ results}

Figure 9 shows the cognitive load of both objective (Task Load Index) and subjective (NASA-TLX) analyzes. The results from the cognitive load assessed by the Task Load Index show that the Hands condition has a significantly higher cognitive load than the Graz one (pairwise paired Wilcox with Bonferroni: $\mathrm{V}=656$, $\mathrm{p}$-value $=0.00063)$. There is no significant difference among the imaginary tasks (TH,RH, LH) and resting state (RS). Meanwhile, the subjective assessment of the cognitive load reflects the opposite. NASA Workload points to a higher cognitive load in the Graz condition instead, although significance could not be found (paired t-test: $\mathrm{t}=0.829$, $\mathrm{p}$ value $=0.428$ ).

Figure 10 shows that the Hands condition presents a non-significant higher Load Magnitude than Graz in factors such as Performance, Physical and Temporal demand. Nevertheless, a pairwise paired Wilcoxon reflects that there is a significant difference between conditions in the Frustration factor $(\mathrm{V}=210$, $\mathrm{p}$-value $=0.049)$, indicating a higher sense of frustration in Graz than Hands condition. 
Finally, a study about the difficulty of performing imaginary tasks was carried out through the Mental Imaginary Questionnaire (MIQ-3). Figure 11 summarizes the user's answers of the MIQ-3 questionnaire, the ratings represent how easy (7) or hard (1) was to perform the imagery task. The mean values show that External Visual Imagery $(5 \pm 1.02)$ was easier for the users than Internal Visual Imagery $(4.8 \pm 1.13)$ and Kinesthetic Imagery $(3.95 \pm 1.24)$.

\section{Discussion}

This study proposed the inclusion of a third arm in an MI-BCI application creating thus a supernumerary limb MI-BCI system. Furthermore, for this approach, the influence of embodiment feedback (Hands) was compared with the standard Graz training in VR. In line with the previous works [34, 35, 26], both the classification rates and the modulation of ERD/ERS signals were enhanced by the realistic feedback, evidencing its importance inside the BCI loop. Also, our work goes further than the one done by Skola and Liarnokapis [34] because they compared an embodied VR scenario against a monitorbased Graz, creating a bias in the users who started with VR. Here, the comparison was made with both Graz and Hands experimental conditions performed in immersive VR.

The presented patterns (Figures 3 and 4) suggest a significantly decreased activity in the sensorimotor area caused by the realistic feedback in comparison with the conventional paradigm ( Graz). Besides this, the ERD activity of TH task is prominent at the three sensorimotor channels $(\mathrm{C} 3, \mathrm{Cz} C 4)$ which could suggest that there is not a compulsory hemisphere governing the control and action of the imaginary third arm. Nevertheless, the analysis of the power changes between tasks (Figures 6 and 7) shows that there are more significant regions at $\mathrm{C} 3$ than at the other electrode positions. This result could indicate that the user's handedness influences the region where TH task presents more activity. In the same way, the common ERD/ERS pattern is visible in $\mathrm{LH}$ and $\mathrm{RH}$ tasks, more in $\mathrm{RH}$ than $\mathrm{LH}$; but it was missing in TH (only an increasing power activity was found in higher frequencies: $>25 \mathrm{~Hz}$ ). It could suggest that the absence of symmetry of the third arm does not elicit a supplementary ERS activity for this task, and this fact is visible in the topographical maps (Figure 5) where the TH in Hands condition presents only ERD activity. This could indicate an effect of the virtual arms support the users to create the abstraction of the third arm. Moreover, 
the unexpected activities presented in the resting state (RS) could be caused by the inertia of the execution/imagery movements. The paradigm to be adopted in the future should include a blank space between the motor task and resting state so that the movements could be easily excluded.

The aim of studying the cognitive load in both subjective and objective ways is for a deeper understanding of the additional load that realistic and visual feedback could cause. In effect, the outcome of the objective assessment (Task Load Index) is not supported by the results of the subjective one (NASA-TLX). EEG data reveals that the cognitive load is higher (significantly) in the realistic condition (Hands) than the standard one (Graz) but the opposite seems to occur in the NASA-TLX (without significance). Moreover, some user's comments at the end of the experiment, such as "I found harder the arrows than the arms" or "I feel Temporal demand a bit easier in Hands than Graz because it is easier to visualize" and the opposite "... The arrow session was a easier than the virtual hands because with the arms I constantly tried to follow the hand movements which did not happen with the arrows" could evidence the disjunctive sensation of the users evidenced by the NASA and Task Load Index. Interestingly, a user did the next comment "The fact that I had the possibility of performing real hand movement helped me to release the stress created by the imagery tasks." This comment supports our decision of keeping the real movements alongside of the imaginary ones, but further studies and comparisons are necessary before drawing conclusions. Finally, the imagery questionnaire shows that the External Visual Imagery was more natural to the users, complementing the comments of the users.

\section{Conclusion and Future work}

This study investigated the possibility of using an imaginary third arm in a BCI system, and shows the differences of the EEG patterns of using a realistic visual training in comparison of the traditional visualization. Initially, the common EEG patterns of motor imagery activity (ERD/ERS) are found when the subjects were asked to imagine a hand movement of a third arm emerging from the chest. These findings can suggest that the illusion of having a third arm could go further than a Rubber Hand illusion since, in this case, a limb is attached and included rather than replaced as RHI does.

In line with the discussion above, the visual processing plays a vital role in the task load. Despite the Hands condition was kept as simple as possible, it 
could not be possible to maintain a low cognitive load like in Graz. In effect, the processing of visual animation is higher than arrows and fixation cross, showing how the visual processing plays a vital role in the task load. However, the benefits presented by this feedback are reflected in the enhancement of the ERD/ERS signals that consequently produces an improvement in the classification. Supernumerary MI-BCI systems are prominent and possible uses should be explored, especially for VR applications, where customized avatars could be controlled using imaginary non-body signals. In effect, Abdi et al. [49] provide evidences about the usefulness and preferences of having three hands in the execution of some activities (i.e. catching objects).

Additionally, and in line with the previous findings done by Skola and Liarnokapis [34], the embodied training improves the classification performance as well as it elicits stronger and consistent ERS/ERD patterns than the traditional Graz paradigm. However, such comparison, unlike that by Skola and Liarnokapis, is done in VR, i.e.; both conditions were made in an immersive VR scenario, eliminating the bias that exists when the comparison is made with Graz in a monitor-based presentation.

An interesting approach would be studying the sense of agency and ownership of the virtual third-arm using both questionnaires or galvanic skin response (GSR), as done by Bashford and Mehring Bashford and Mehring [38]. This would provide a wider body of knowledge about the use of a supernumerary BCI system. Besides this, an online experiment is mandatory to validate the initial results as well as studies about the handedness of the third arm using left-handed subjects.

Finally, this work also intends to provide premises regarding the role of mental imagery in the exploration of cognitive processes. If we look at the present work from the perspective of embodied cognition, we can argue that supernumerary BCI systems can allow us to study the human ability for body extrapolation and how the mind can be shaped by these new experiences. A discussion is open towards the use of imaginary limbs as a means to control system, extending the human mind constraints imposed by the body.

\section{Acknowledgements}

This study was partly funded by the Coordenação de Aperfeiçoamento de Pessoal de Nível Superior - Brasil (CAPES) - Finance Code 001, and partly by CNPq. We also acknowledge FAPERGS (project 17/2551-0001192-9) and CNPq-Brazil (project 311353/2017-7) for their financial support. Special 
thanks are also due to Petrobras for the support through the Annelida research project. The first author would like to thank to the SDAS Research Group (www.sdas-group.com) for its valuable support.

\section{References}

[1] C. Neuper, G. Muller, A. Kubler, N. Birbaumer, G. Pfurtscheller, Clinical application of an eeg-based brain-computer interface: a case study in a patient with severe motor impairment, Clinical Neurophysiology 114 (2003) $399-409$.

[2] C. Neuper, G. Pfurtscheller, Neurofeedback Training for BCI Control, Springer Berlin Heidelberg, Berlin, Heidelberg, pp. 65-78.

[3] T. O. Zander, C. Kothe, Towards passive braincomputer interfaces: applying braincomputer interface technology to humanmachine systems in general, Journal of Neural Engineering 8 (2011) 025005.

[4] T. O. Zander, C. Kothe, S. Jatzev, M. Gaertner, Enhancing HumanComputer Interaction withInput from Active and Passive BrainComputer Interfaces, Springer London, London, pp. 181-199.

[5] T. O. Zander, M. Gaertner, C. Kothe, R. Vilimek, Combining eye gaze input with a braincomputer interface for touchless humancomputer interaction, International Journal of HumanComputer Interaction 27 (2010) 38-51.

[6] E. Donchin, K. M. Spencer, R. Wijesinghe, The mental prosthesis: assessing the speed of a p300-based brain-computer interface, IEEE Transactions on Rehabilitation Engineering 8 (2000) 174-179.

[7] M. Jeannerod, Mental imagery in the motor context, Neuropsychologia 33 (1995) 1419 - 1432. The Neuropsychology of Mental Imagery.

[8] J. R. Wolpaw, N. Birbaumer, D. J. McFarland, G. Pfurtscheller, T. M. Vaughan, Braincomputer interfaces for communication and control, Clinical Neurophysiology 113 (2002) 767 - 791.

[9] G. Pfurtscheller, C. Neuper, Motor imagery and direct brain-computer communication, Proceedings of the IEEE 89 (2001) 1123-1134. 
[10] P. Gert, R. Leeb, J. Faller, C. Neuper, Brain-computer interface systems used for virtual reality control, in: J.-J. Kim (Ed.), Virtual Reality, InTech, 2011, pp. 1-19.

[11] R. Chavarriaga, M. Fried-Oken, S. Kleih, F. Lotte, R. Scherer, Heading for new shores! overcoming pitfalls in bci design, Brain Computer Interfaces (Abingdon, England) 4 (2017) 60-73.

[12] F. Lotte, L. Bougrain, A. Cichocki, M. Clerc, M. Congedo, A. Rakotomamonjy, F. Yger, A review of classification algorithms for eeg-based braincomputer interfaces: a 10 year update, Journal of Neural Engineering 15 (2018) 031005.

[13] F. Lotte, C. Jeunet, Defining and quantifying users' mental imagerybased BCI skills: a first step, Journal of Neural Engineering 15 (2018) 046030.

[14] F. Lotte, C. Jeunet, Online classification accuracy is a poor metric to study mental imagery-based bci user learning: an experimental demonstration and new metrics, in: 7th International BCI Conference, Graz, Austria.

[15] F. Lotte, C. Jeunet, J. Mladenovic, B. N'Kaoua, L. Pillette, A BCI challenge for the signal processing community: considering the user in the loop, in: Signal Processing and Machine Learning for Brain-Machine Interfaces, IET, 2018, pp. 1-33. Chapter 8.

[16] A. HA, Virtual reality programs applications in healthcare, Health Med Informat 9 (2018) 305.

[17] C. Donalek, S. G. Djorgovski, A. Cioc, A. Wang, J. Zhang, E. Lawler, S. Yeh, A. Mahabal, M. Graham, A. Drake, S. Davidoff, J. S. Norris, G. Longo, Immersive and collaborative data visualization using virtual reality platforms, in: 2014 IEEE International Conference on Big Data (Big Data), pp. 609-614.

[18] P. Gamito, J. Oliveira, C. Coelho, D. Morais, P. Lopes, J. Pacheco, R. Brito, F. Soares, N. Santos, A. F. Barata, Cognitive training on stroke patients via virtual reality-based serious games, Disability and Rehabilitation 39 (2017) 385-388. 
[19] A. Lcuyer, F. Lotte, R. B. Reilly, R. Leeb, M. Hirose, M. Slater, Braincomputer interfaces, virtual reality, and videogames, Computer 41 (2008) 66-72.

[20] E. C. Lalor, S. P. Kelly, C. Finucane, R. Burke, R. Smith, R. B. Reilly, G. McDarby, Steady-state vep-based brain-computer interface control in an immersive 3d gaming environment, EURASIP Journal on Advances in Signal Processing 2005 (2005) 706906.

[21] R. Leeb, D. Friedman, G. R. Mller-Putz, R. Scherer, M. Slater, G. Pfurtscheller, Self-paced (asynchronous) bci control of a wheelchair in virtual environments: A case study with a tetraplegic, Computational Intelligence and Neuroscience 79642 (2007).

[22] F. Lotte, Y. Renard, A. Lécuyer, Self-Paced Brain-Computer Interaction with Virtual Worlds: A Quantitative and Qualitative Study "Out of the Lab", in: 4th international Brain Computer Interface Workshop and Training Course, Graz University of Technology, Graz, Austria.

[23] M. Botvinick, J. Cohen, Rubber hands feel touch that eyes see, Nature 391 (1998) $756 \mathrm{EP}$-.

[24] F. Ferri, A. M. Chiarelli, A. Merla, V. Gallese, M. Costantini, The body beyond the body: expectation of a sensory event is enough to induce ownership over a fake hand, Proceedings of the Royal Society B: Biological Sciences 280 (2013) 20131140.

[25] A. Kalckert, H. Ehrsson, Moving a rubber hand that feels like your own: A dissociation of ownership and agency, Frontiers in Human Neuroscience 6 (2012) 40.

[26] J. Riascos, S. Villa, A. Maciel, L. Nedel, D. Barone, Towards moving virtual arms using brain-computer interface, in: M. Gavrilova, J. Chang, N. M. Thalmann, E. Hitzer, H. Ishikawa (Eds.), Advances in Computer Graphics, Springer International Publishing, Cham, 2019, pp. 445-452.

[27] S. G. Hart, L. E. Stavenland, Development of NASA-TLX (Task Load Index): Results of empirical and theoretical research, in: P. A. Hancock, N. Meshkati (Eds.), Human Mental Workload, Elsevier, 1988, pp. 139183. 
[28] M. E. Smith, A. Gevins, H. Brown, A. Karnik, R. Du, Monitoring task loading with multivariate eeg measures during complex forms of human-computer interaction, Human Factors 43 (2001) 366-380.

[29] P. Mendes, D. A. Marinho, J. Petrica, P. Silveira, D. Monteiro, L. Cid, Translation and validation of the movement imagery questionnaire 3 (miq - 3) with portuguese athletes, Motricidade 12 (2016) 149.

[30] C. Neuper, G. Pfurtscheller, Neurofeedback Training for BCI Control, Springer Berlin Heidelberg, Berlin, Heidelberg, pp. 65-78.

[31] F. Lotte, J. Faller, C. Guger, Y. Renard, G. Pfurtscheller, A. Lécuyer, R. Leeb, Combining BCI with Virtual Reality: Towards New Applications and Improved BCI, in: B. Z. Allison, S. Dunne, R. Leeb, J. D. R. Millán, A. Nijholt (Eds.), Towards Practical Brain-Computer Interfaces:, Springer, 2013.

[32] C. Neuper, R. Scherer, S. Wriessnegger, G. Pfurtscheller, Motor imagery and action observation: Modulation of sensorimotor brain rhythms during mental control of a brain-computer interface, Clinical Neurophysiology 120 (2009) $239-247$.

[33] R. Ron-Angevin, A. Diaz-Estrella, Brain-computer interface: Changes in performance using virtual reality techniques, Neuroscience Letters 449 (2009) $123-127$.

[34] F. Skola, F. Liarokapis, Embodied vr environment facilitates motorimagery brain-computer interface training, Computers and Graphics 75 (2018) $59-71$.

[35] N. Braun, R. Emkes, J. D. Thorne, S. Debener, Embodied neurofeedback with an anthropomorphic robotic hand, Scientific Reports 6 (2016) $37696 \mathrm{EP}$-. Article.

[36] M. Alimardani, S. Nishio, H. Ishiguro, Effect of biased feedback on motor imagery learning in bci-teleoperation system, Frontiers in Systems Neuroscience 8 (2014) 52 .

[37] M. Alimardani, S. Nishio, H. Ishiguro, Removal of proprioception by bci raises a stronger body ownership illusion in control of a humanlike robot, Scientific Reports 6 (2016) 33514 EP -. Article. 
[38] L. Bashford, C. Mehring, Ownership and agency of an independent supernumerary hand induced by an imitation brain-computer interface, PLOS ONE 11 (2016) 1-15.

[39] M. Song, J. Kim, A paradigm to enhance motor imagery using rubber hand illusion induced by visuo-tactile stimulus, IEEE Transactions on Neural Systems and Rehabilitation Engineering 27 (2019) 477-486.

[40] C. Neuper, R. Scherer, M. Reiner, G. Pfurtscheller, Imagery of motor actions: Differential effects of kinesthetic and visualmotor mode of imagery in single-trial eeg, Cognitive Brain Research 25 (2005) 668 677.

[41] M. xfleckx, Lsl4unity: A integration approach of the labstreaminglayer framework for unity3d, 2019.

[42] A. Delorme, S. Makeig, Eeglab: an open source toolbox for analysis of single-trial eeg dynamics including independent component analysis, Journal of Neuroscience Methods 134 (2004) 9 - 21.

[43] R. Leeb, C. Keinrath, D. Friedman, C. Guger, R. Scherer, C. Neuper, M. Garau, A. Antley, A. Steed, M. Slater, G. Pfurtscheller, Walking by thinking: The brainwaves are crucial, not the muscles!, Presence 15 (2006) 500-514.

[44] A. Delorme, S. Makeig, Eeglab: an open source toolbox for analysis of single-trial eeg dynamics including independent component analysis, Journal of Neuroscience Methods 134 (2004) $9-21$.

[45] R. Roberts, N. Callow, L. Hardy, D. Markland, J. Bringer, Movement imagery ability: Development and assessment of a revised version of the vividness of movement imagery questionnaire, Journal of sport and exercise psychology 30 (2008) 200-21.

[46] F. Atienza, I. Balaguer, M. L. Garcia-Merita, Factor analysis and reliability of the movement imagery questionnaire, Perceptual and Motor Skills 78 (1994) 1323-1328.

[47] S. Williams, J. Cumming, N. Ntoumanis, S. M Nordin-Bates, R. Ramsey, C. Hall, Further validation and development of the movement imagery questionnaire, Journal of sport and exercise psychology 34 (2012) $621-46$. 
687

688

689

[48] P. A. Mendes, D. A. Marinho, J. D. Petrica, P. Silveira, D. Monteiro, L. Cid, Traduo e validao do movement imagery questionnaire - 3 (miq - 3) com atletas portugueses, Motricidade 12 (2016) $149-158$.

[49] E. Abdi, E. Burdet, M. Bouri, S. Himidan, H. Bleuler, In a demanding task, three-handed manipulation is preferred to two-handed manipulation, Scientific Reports 6 (2016) 21758 EP -. Article. 
bioRxiv preprint doi: https://doi.org/10.1101/817205; this version posted October 24, 2019. The copyright holder for this preprint (which was not certified by peer review) is the author/funder, who has granted bioRxiv a license to display the preprint in perpetuity. It is made available under aCC-BY-NC-ND 4.0 International license.

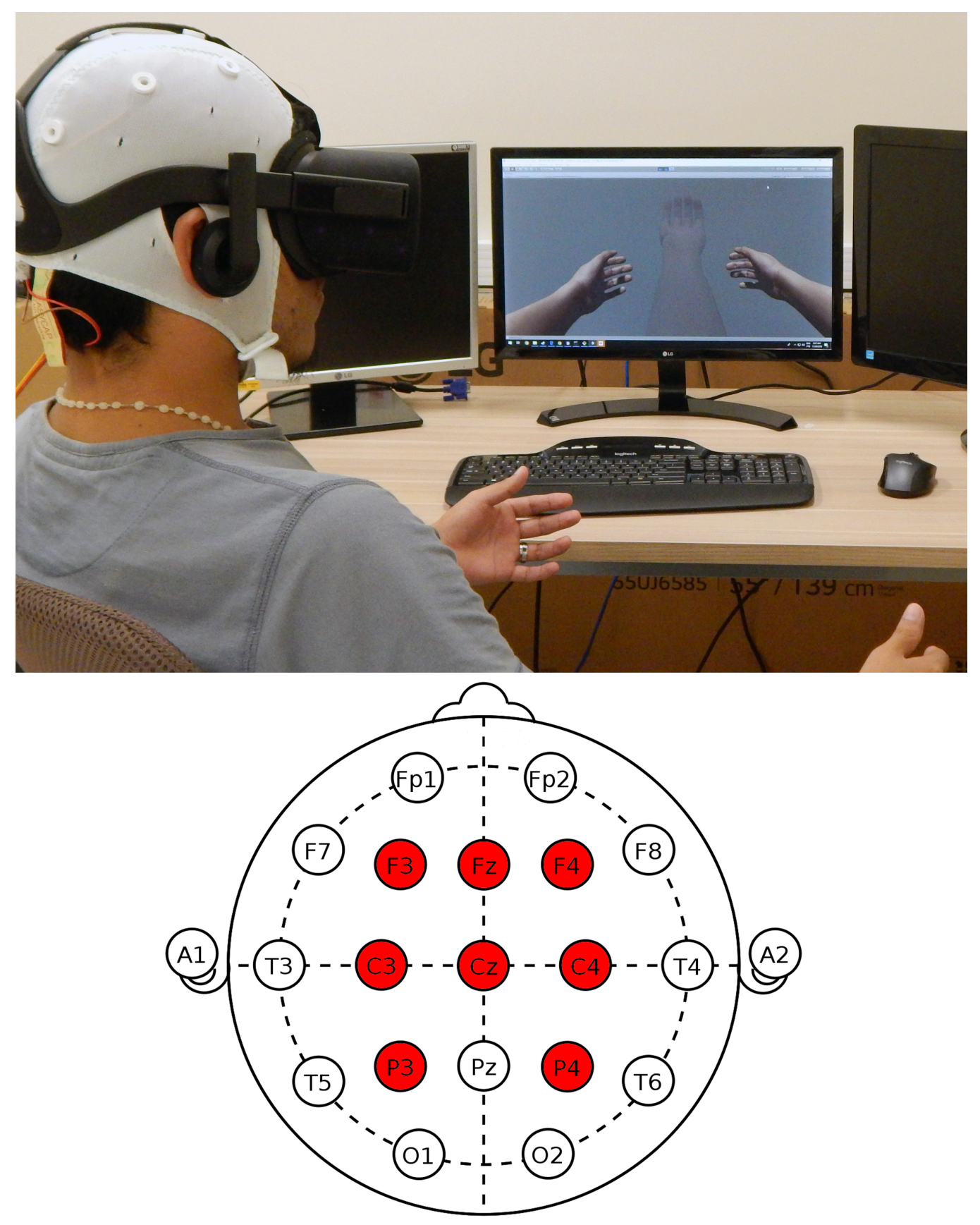

Figure 1: Experiment setup: A subject using a BCI interface to control his "three" arms in a virtual reality experience (top); and the electrodes placement over the sensorimotor area (filled circle), following the 10-20 system (bottom). 


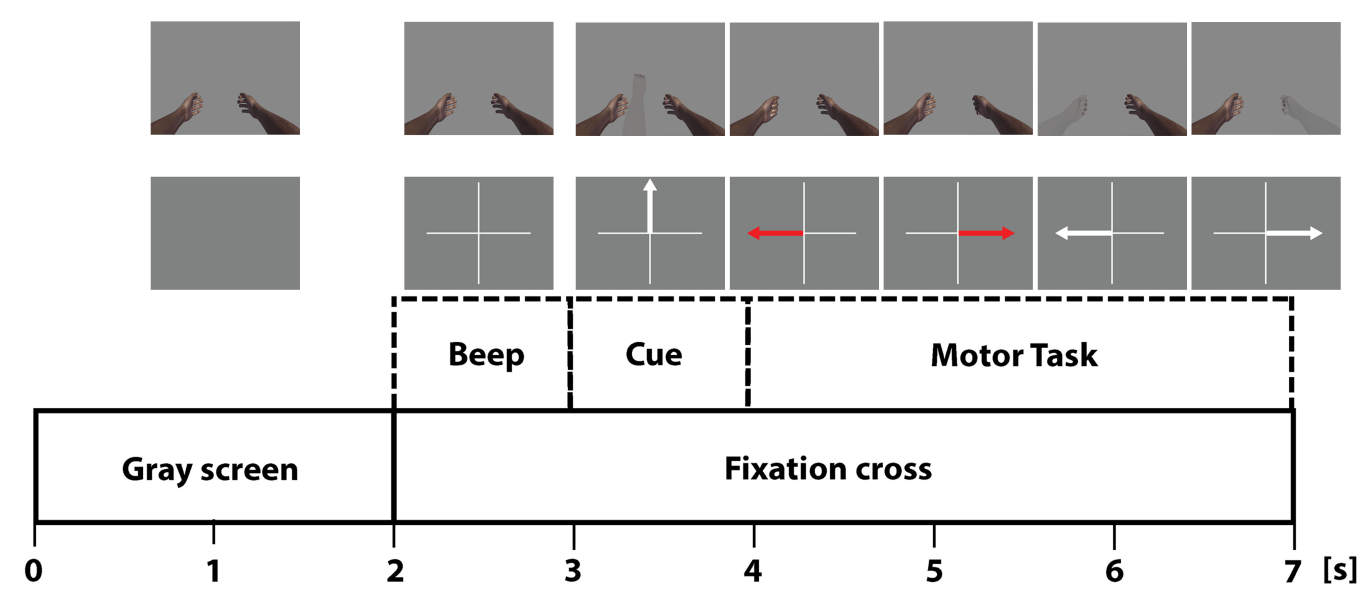

Figure 2: Experiment paradigm. The visual stimulus of the task's cue are corresponding for both conditions. Top: visual stimuli for Hands condition. Middle: visual stimuli for Graz condition. Bottom: timing of the trials following the classic Graz protocol. 
bioRxiv preprint doi: https://doi.org/10.1101/817205; this version posted October 24, 2019. The copyright holder for this preprint (which was not certified by peer review) is the author/funder, who has granted bioRxiv a license to display the preprint in perpetuity. It is made available under aCC-BY-NC-ND 4.0 International license.
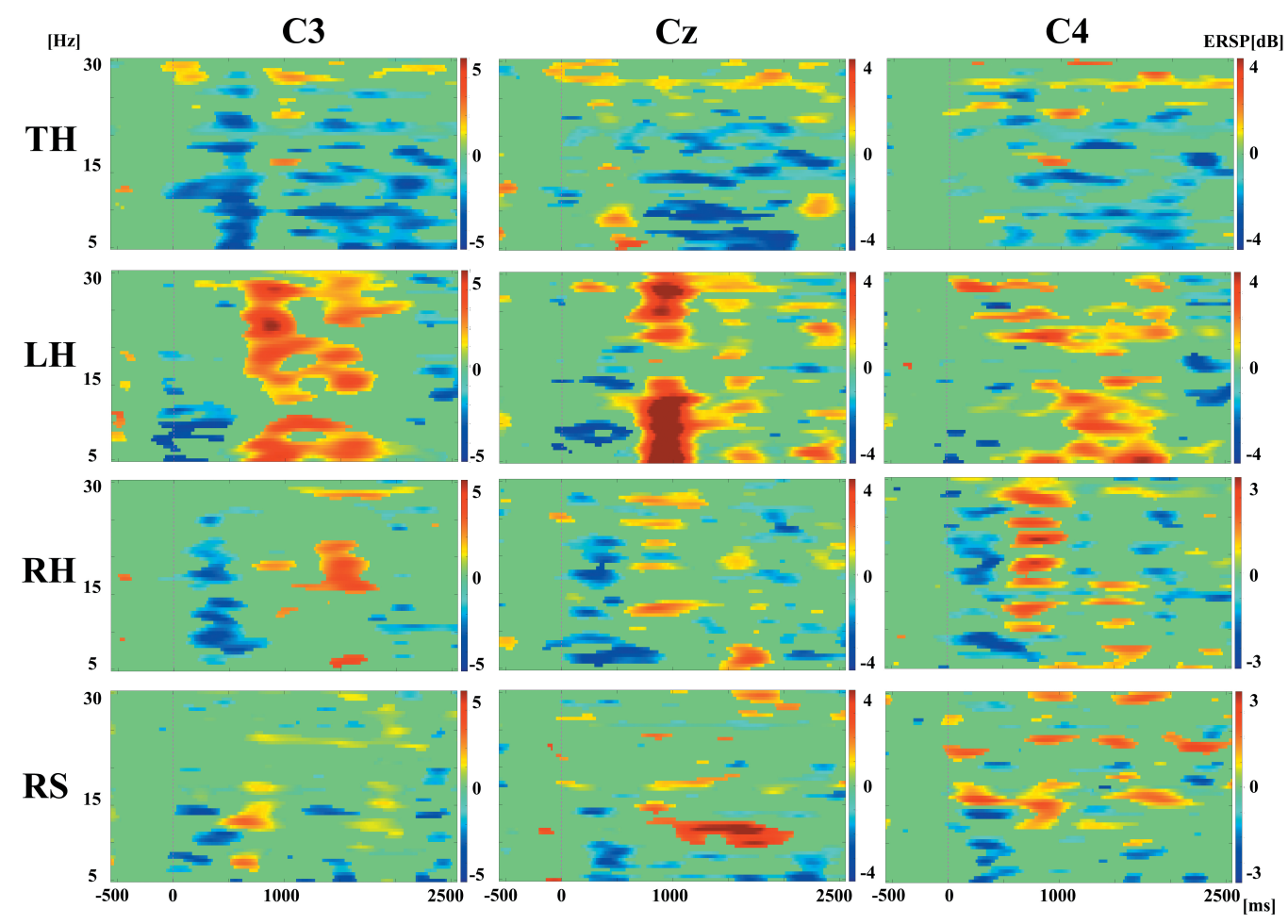

Figure 3: Significant ERD/ERS patterns of the mental task at $\mathrm{C} 3, \mathrm{Cz}, \mathrm{C} 4$ positions for Hands condition (blue indicates ERD). A strong ERD activity is found at the three electrodes for the third hand (TH). Whereas, ERS patterns are found mainly for the left hand $(\mathrm{LH})$. The ERD/ERS fluctuation is more visible for the right hand (RH), mostly at C4. 

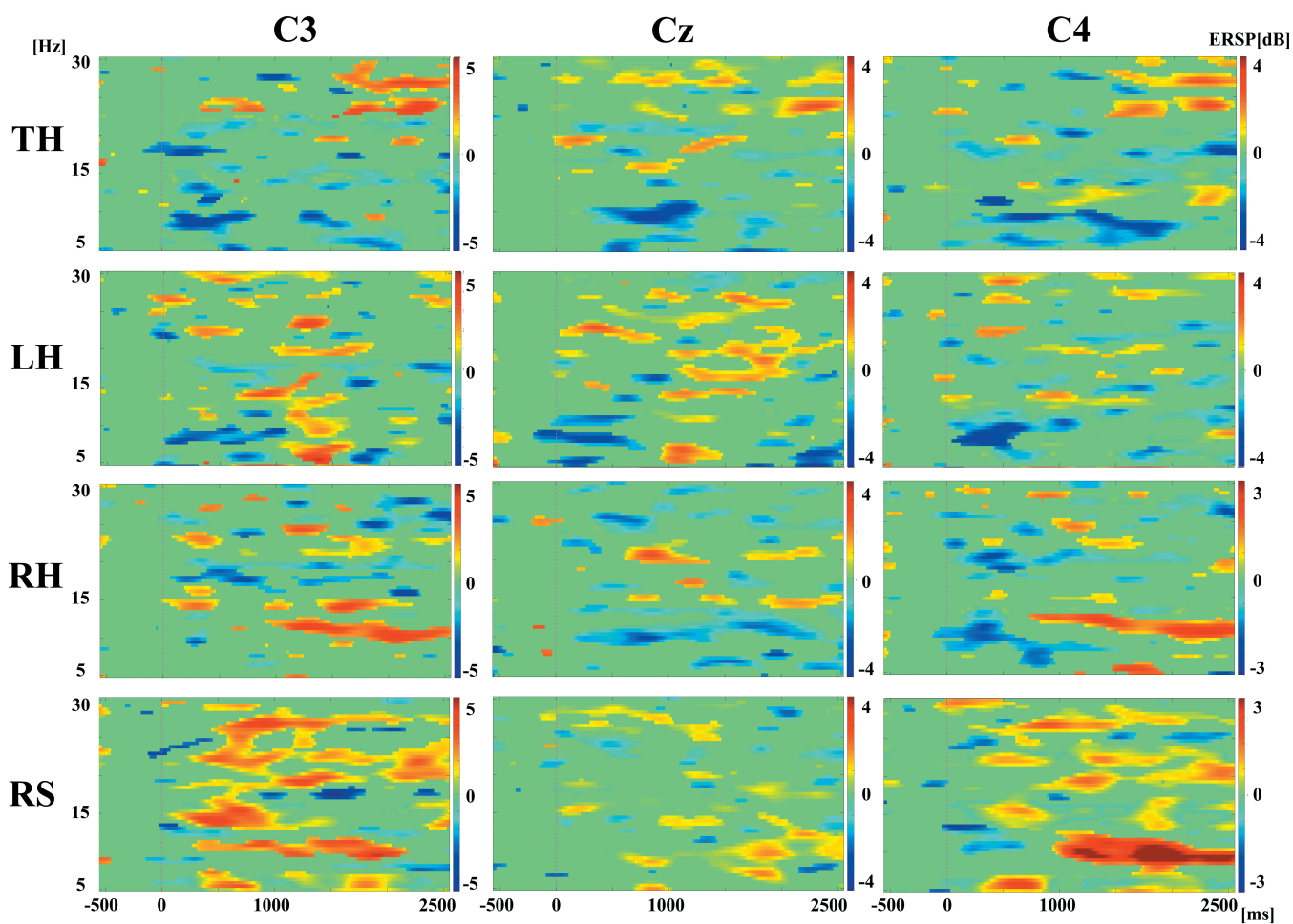

Figure 4: Significant ERD/ERS patterns of the mental task at C3, Cz, C4 positions for Graz condition (blue indicates ERD). An ERD activity is mainly found in the alpha band $(8-12 \mathrm{~Hz})$ at the three electrodes for the third hand $(\mathrm{TH})$. The ERD/ERS patterns are widespread for left and right hands (LH, RH respectively) at the three electrodes. There is extensive activity in the resting state (RS).

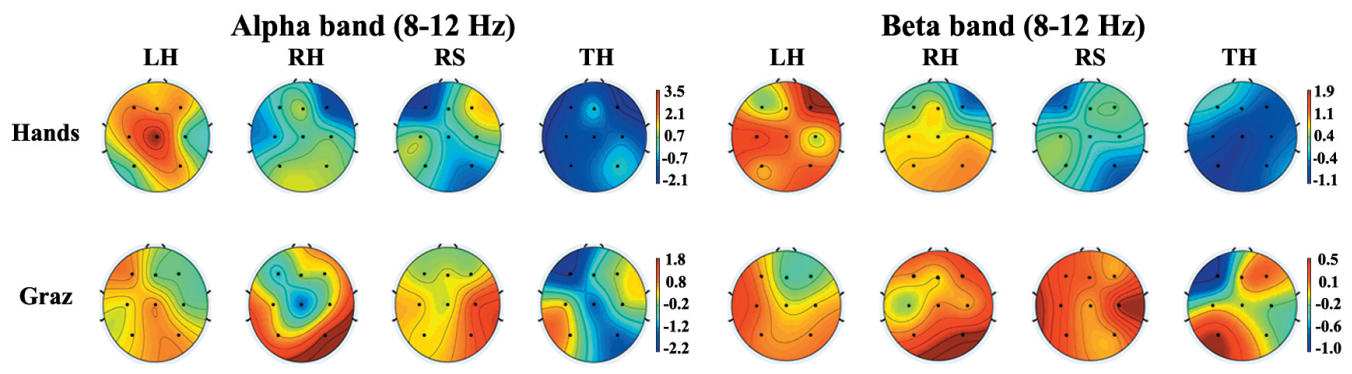

Figure 5: Topographical distribution of each task for both conditions (blue indicates ERD). The maps are made using the ERSP values in both Alpha and Beta bands, one second after the cue. Blue indicates the ERD activity during the mental tasks. 

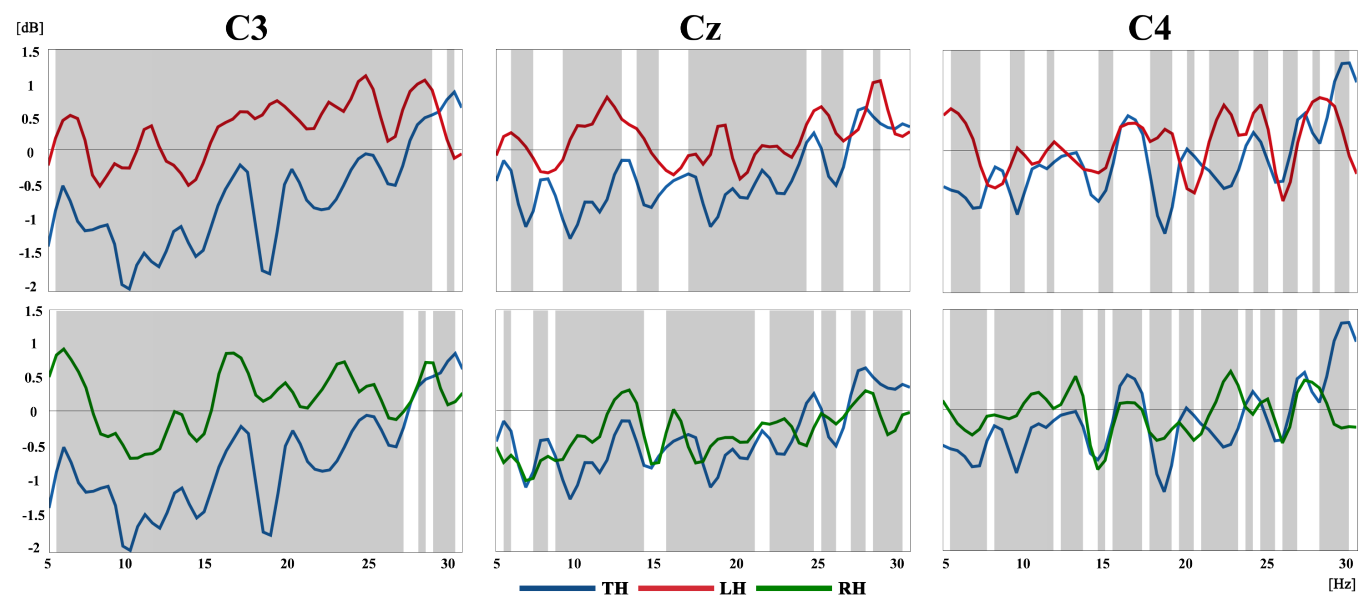

Figure 6: Comparison of the power changes of the mental tasks in the sensory-motor area (C3, Cz, C4) in Hands condition. Top: Third hand (TH, blue) - Left hand (LH, red). Bottom: Third hand (TH, blue) - Right hand (RH, red).
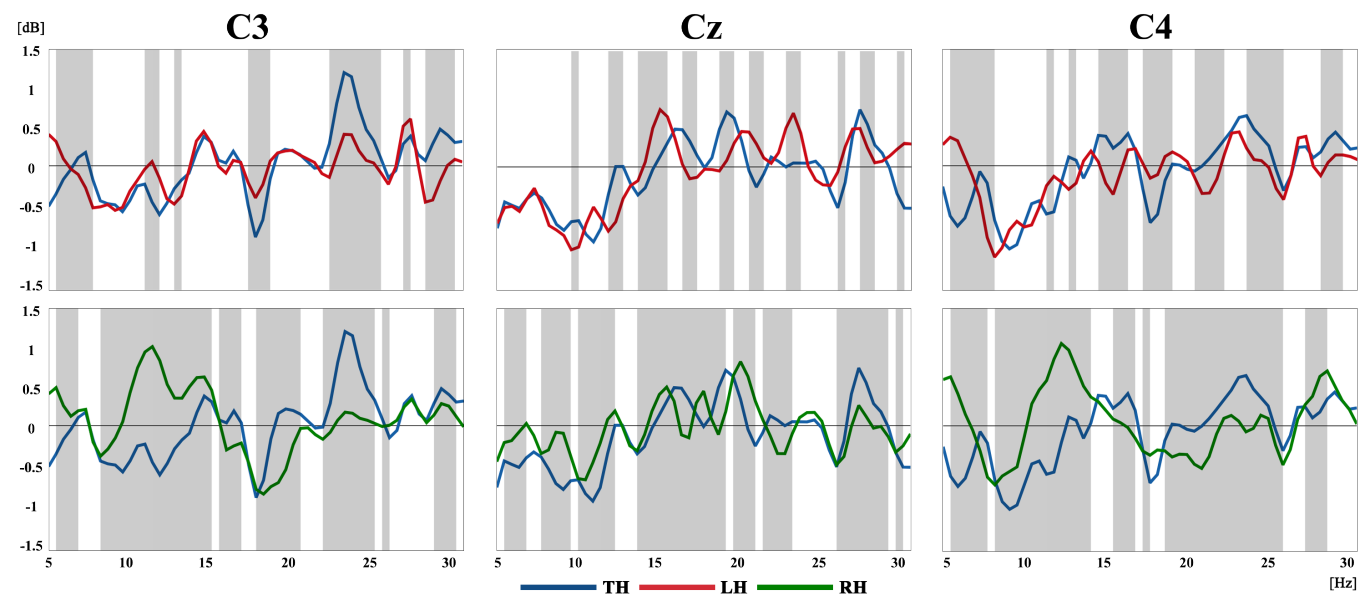

Figure 7: Comparison of the power changes of the mental tasks in the sensory-motor area (C3, Cz, C4) in Graz condition. Top: Third hand (TH, blue) - Left hand (LH, red). Bottom: Third hand ( $\mathrm{TH}$, blue) - Right hand ( $\mathrm{RH}$, red). 

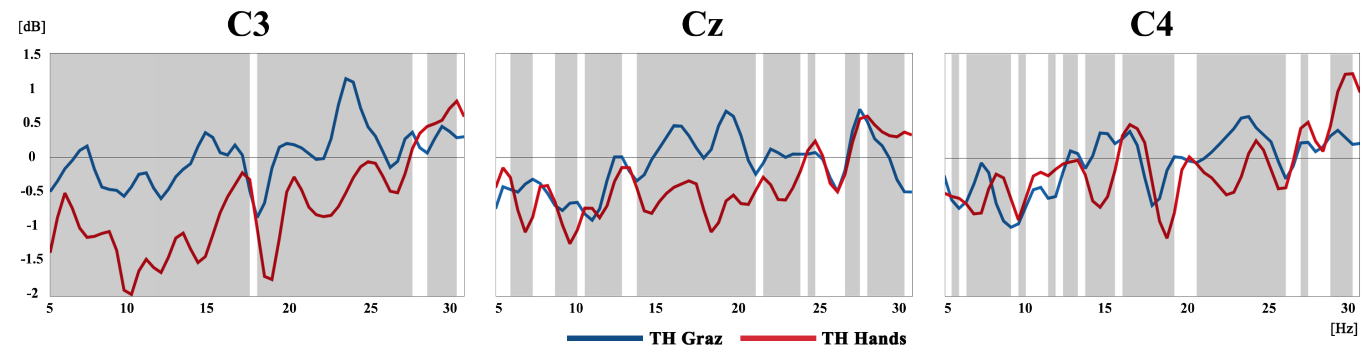

Figure 8: Comparison of the power changes of the third arm task in the sensory-motor area (C3, Cz, C4) for both conditions. Blue: Third hand in Graz condition. Red: Third hand in Hands condition.

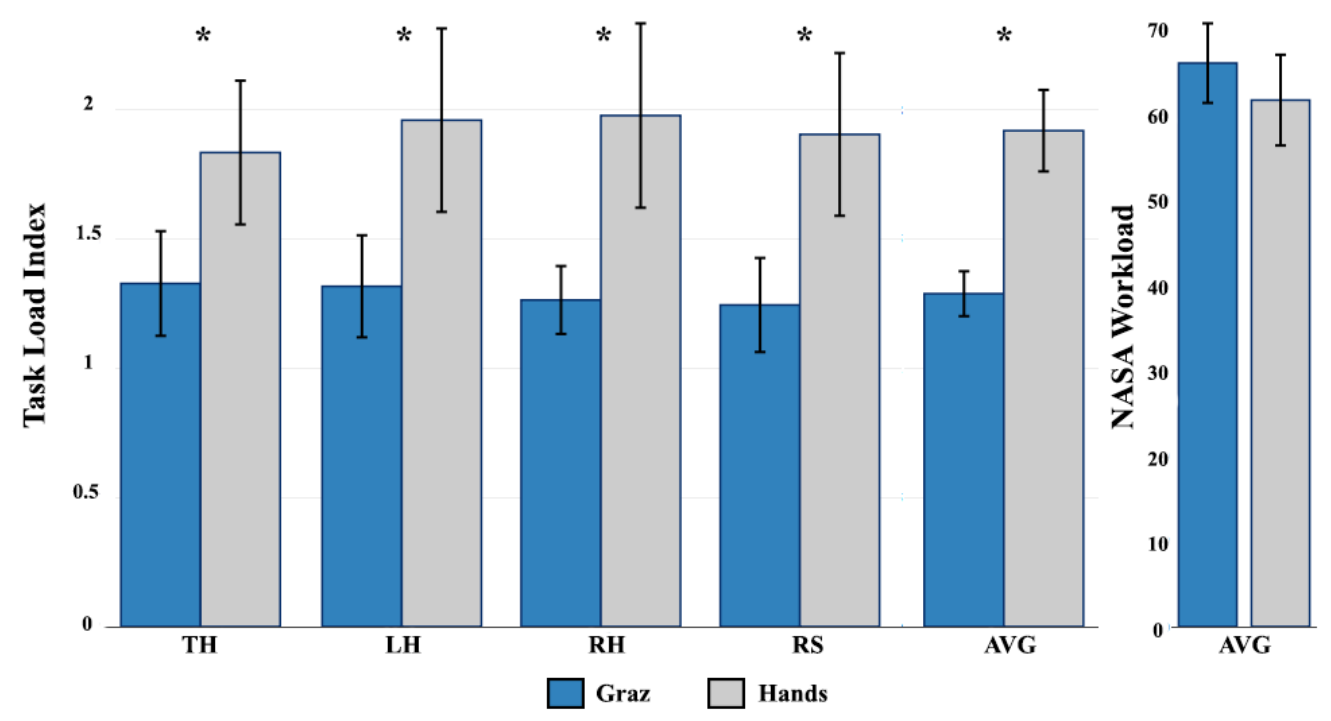

Figure 9: Task Load Index and NASA Workload assessment for the two conditions. * Significant differences 
bioRxiv preprint doi: https://doi.org/10.1101/817205; this version posted October 24, 2019. The copyright holder for this preprint (which was not certified by peer review) is the author/funder, who has granted bioRxiv a license to display the preprint in perpetuity. It is made available under aCC-BY-NC-ND 4.0 International license.

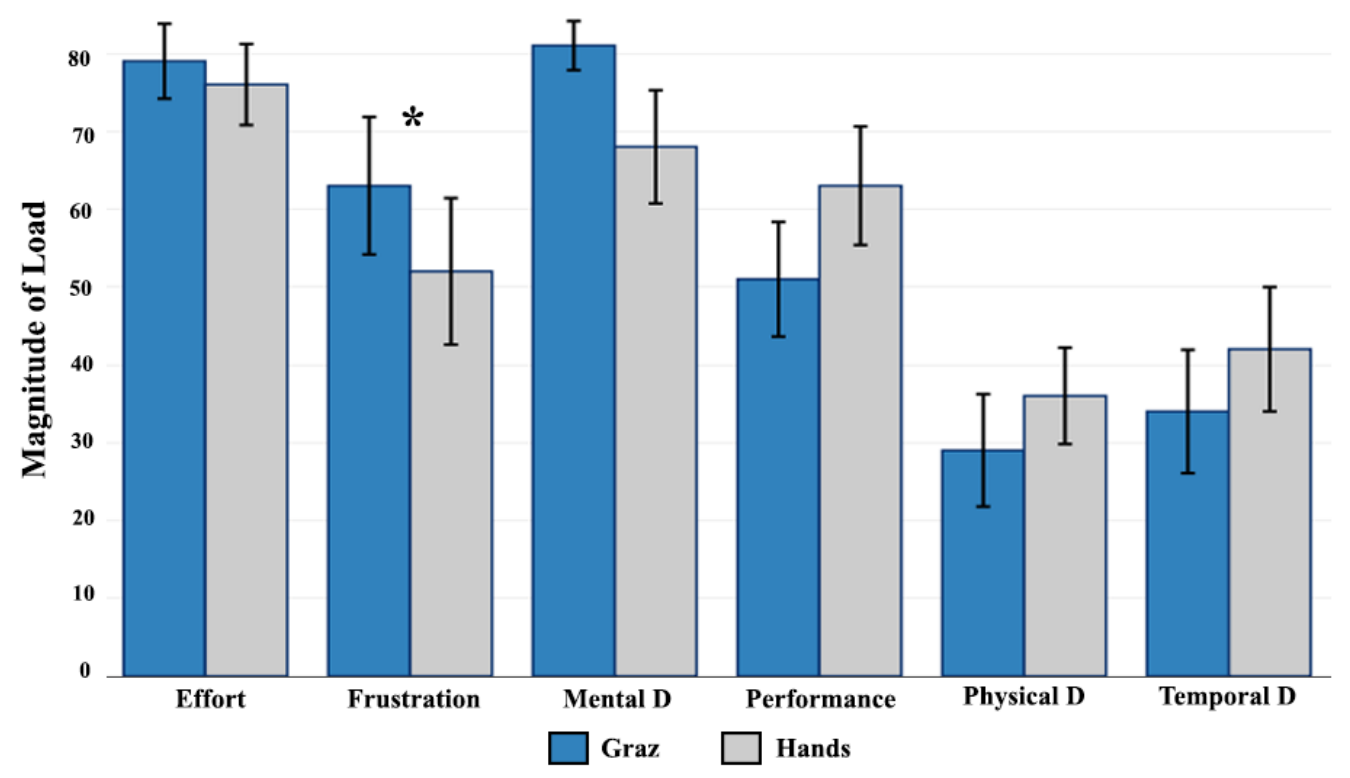

Figure 10: NASA factors for the two conditions. ${ }^{*}$ Significant difference.

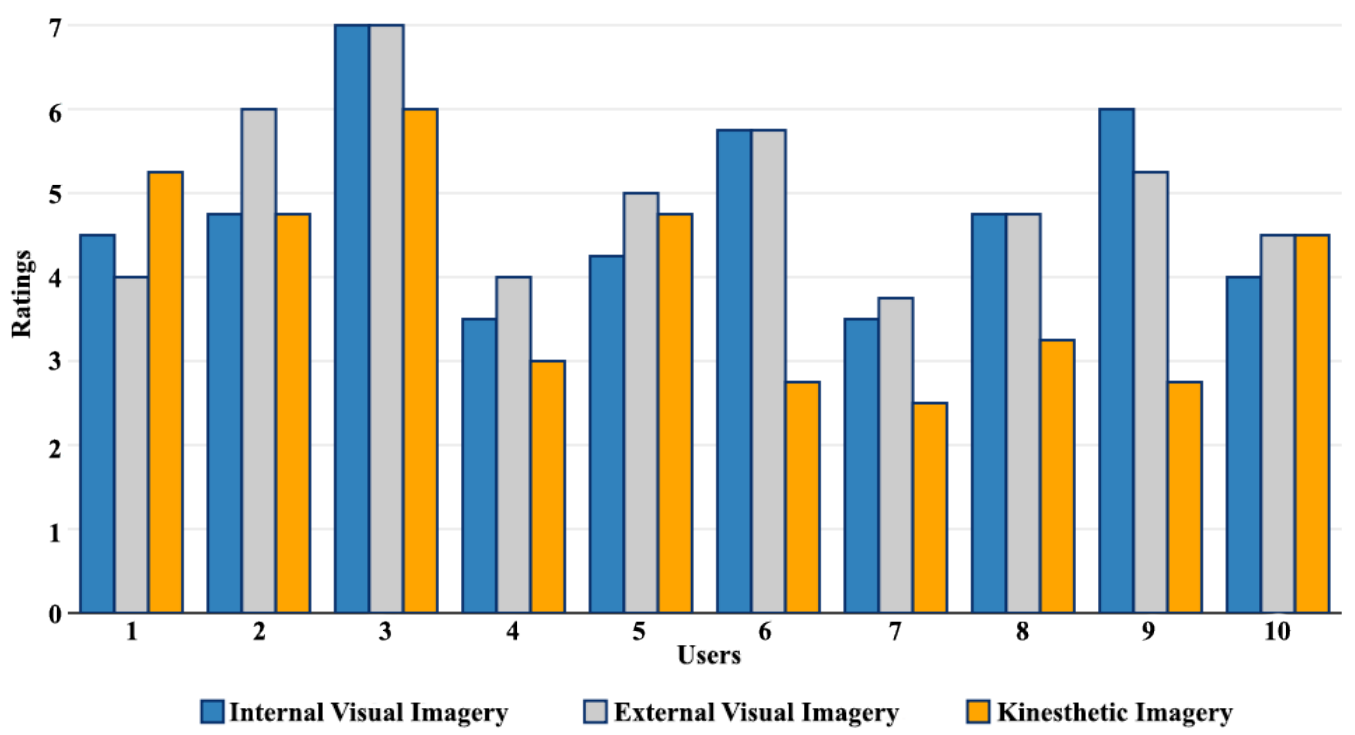

Figure 11: MIQ-3 results. Ratings range from 1 (very hard) to 7 (very easy). 